\title{
PAPER
}

\section{Second harmonic imaging: a new ultrasound technique to assess human brain tumour perfusion}

\author{
J U Harrer, L Mayfrank, M Mull, C Klöłzsch
}

J Neurol Neurosurg Psychiatry 2003;74:333-338

See end of article for authors' affiliations

.....................

Correspondence to: Dr med J Harrer, Department of Neurology,

University Hospital Aachen

Pauwelsstr 30, D-52074

Aachen, Germany;

judith.harrer@post.rwth-

aachen.de

Received 7 August 2002

In revised form 5

September 2002

Accepted

11 November 2002
Background: Second harmonic imaging is a new ultrasound technique that allows evaluation of brain tissue perfusion after application of an ultrasound contrast agent.

Objective: To evaluate the potential of this technique for the assessment of abnormal echo contrast characteristics of different brain tumours.

Methods: 27 patients with brain tumours were studied. These were divided into four groups: gliomas, WHO grade III-IV $(n=6)$; meningiomas $(n=9)$; metastases $(n=5)$; and others $(n=7)$. Patients were examined by second harmonic imaging in a transverse axial insonation plane using the transtemporal approach. Following intravenous administration of $4 \mathrm{~g}(400 \mathrm{mg} / \mathrm{ml})$ of a galactose based echo contrast agent, 62 time triggered images (one image per 2.5 seconds) were recorded and analysed offline. Time-intensity curves of two regions of interest (tumour tissue and healthy brain tissue), including peak intensity $(P I)(d B)$, time to peak intensity (TP) (s), and positive gradient (PG) $(d B / s)$, as well as ratios of the peak intensities of the two regions of interest, were derived from the data and compared intraindividually and interindividually.

Results: After administration of the contrast agent a marked enhancement of echo contrast was visible in the tumour tissue in all patients. Mean PI and PG were significantly higher in tumour tissue than in healthy brain parenchyma $(11.8 \vee 5.1 \mathrm{~dB}$ and $0.69 \vee 0.16 \mathrm{~dB} / \mathrm{s} ; \mathrm{p}<0.001)$. TP did not differ significantly (37.1 v $50.2 \mathrm{~s} ; \mathrm{p}=0.14)$. A tendency towards higher PI and PG as well as shorter TP was apparent in malignant gliomas. When comparing different tumour types, however, none of these variables reached significance, nor were there significant differences between malignant and benign tumours in general.

Conclusions: Second harmonic imaging not only allows identification of brain tumours, but may also help in distinguishing between different tumour types. It gives additional and alternative information about tumour perfusion. Further studies are needed to evaluate the clinical potential of this technique in investigating brain tumours - for example in follow up investigations of patients undergoing radiation or chemotherapy-especially in comparison with neuroradiological and neuropathological findings.
$\mathrm{T}$ he development of microencapsulated gas bubble contrast agents possessing strongly non-linear properties led to the introduction of second harmonic imaging (SHI) in the early $1990 \mathrm{~s}^{1-3}$ Since then, SHI has proved to be a useful diagnostic tool for demonstrating and evaluationg perfusion disturbances, especially in myocardial and hepatic tissue. ${ }^{4-7}$ Several recent reports have demonstrated the potential of transcranial SHI for assessing brain tissue perfusion in healthy volunteers as well as perfusion disturbances in acute ischaemic stroke. - $^{8-12}$

On the basis of the non-linear properties of ultrasound contrast agents, SHI differentiates echoes from microbubbles in the capillary bed from those of the avascular tissue. While insonated tissue responds primarily at the fundamental frequency, resonating microbubbles cause scattering of multiples of the fundamental frequency-the harmonic frequencies. By the use of a filter set to receive preferentially the echoes from the microbubbles and to suppress those from solid tissues, harmonic frequencies can be selected for image acquisition. As a result the signal to noise ratio is dramatically increased and perfusion (blood flow in small vessels) is not only detectable but also assessable. ${ }^{1-4}$ Acoustic densitometry allows analysis of the average acoustic intensity and calculation of time-intensity curves of a series of contrast enhanced harmonic images in user specified regions of interest (ROI). ${ }^{13}$

Ultrasonography has been used for over 40 years for the detection and follow up investigation of tumour growth and dissemination. ${ }^{14}$ Liver lesions are especially sensitive to detec- tion by ultrasound. Application of microbubbles in combination with microbubble specific imaging modes, such as second harmonic imaging and pulse inversion harmonic imaging, have simplified detection and improved the characterisation of several hepatic tumours by their characteristic features and haemodynamics in comparison with conventional Doppler ultrasound or grey scale imaging..${ }^{14-18}$

Up to now, the non-invasive diagnosis of human brain tumours has usually involved neuroradiological imaging techniques including computed tomography (CT), magnetic resonance imaging (MRI), and magnetic resonance spectroscopy, as well as positron emission tomography (PET) and single photon emission computed tomography (SPECT). $.^{19-22} \mathrm{Al}-$ though new magnetic resonance techniques are particularly sensitive, they are not specific, and further histological assessment is often required to reach a definitive diagnosis. New imaging techniques that are intended to specify the histological grade of a tumour analyse the pathophysiology rather than the structure of a tumour. Apart from radionuclide based techniques, perfusion MRI has been shown to have a high sensitivity and specificity for tumour detection and noninvasive tumour grading. ${ }^{23}{ }^{24}$ However, these techniques can be stressful or even intolerable for the critically ill patient. This is particularly important when frequent follow up examinations

Abbreviations: $P G$, positive gradient; $\mathrm{PI}$, peak intensity; $\mathrm{ROI}$, region of interest; SHI, second harmonic imaging; TP, time to peak intensity 
are needed. Moreover, they are both time consuming and expensive.

Transcranial ultrasound investigation has been of limited value for the diagnostics of brain tumours in the past. The detection rate of brain tumours with conventional transcranial sonography lies between $40 \%$ and $80 \%$, depending on the tumour type, the quality of the acoustic window, and whether or not the tumour localisation is known to the investigator through other neuroradiological imaging modes. ${ }^{25}$ It has already been shown that for planning non-invasive treatment and for postoperative follow up of brain tumours, CT and MRI may well be complemented by transcranial sonography. ${ }^{26-28}$

Second harmonic imaging is the first ultrasound technique allowing assessment of brain tissue perfusion. Previous studies have shown its high potential not only to analyse capillary blood flow in healthy individuals but also to assess perfusion deficits in acute ischaemic infarction. ${ }^{8-11}$ Our aim in the present study was to evaluate the clinical potential of SHI for visualising and quantifying brain tumour perfusion. We aimed to correlate various sonographic perfusion indices with degrees of vascularisation of different tumour types. In addition, we sought to investigate whether SHI allows discrimination between benign and malignant tumours.

\section{METHODS \\ Patients}

Between January and September 2001 SHI was undertaken on 27 adult inpatients with untreated brain tumours of any type. These included gliomas of WHO grade II-IV, oligodendrogliomas, neurilemmomas, meningiomas, tumours of the pituitary gland, chordomas, and metastases. Tumour diagnosis was made from neuroradiological and histopathological findings. Of these patients, 15 had a benign tumour and 12 a malignant tumour. Their mean age was 56 years (range 28 to 77 years; 18 male, nine female). Inclusion criteria for the patients were that they were aged over 18 and had an adequate acoustic bone window enabling the depiction of the brain stem and third ventricle in native B mode imaging. Exclusion criteria were galactosaemia, pregnancy or lactation, a history of alcohol or drug abuse, and previous allergic reactions to the contrast agent. All patients had a complete physical and neurological examination, routine blood tests, and a 12 lead ECG.

Informed consent for the procedure, including the administration of an ultrasound contrast agent, was obtained from all the patients.

\section{Technical equipment and ultrasound contrast agent}

All examinations were carried out with the Hewlett-Packard Sonos 5500® duplex device (Hewlett-Packard Inc, Palo Alto, California, USA) coupled with a 1.8/3.6 MHz sector transducer (S3 probe, Hewlett-Packard) capable of fundamental and harmonic imaging. All harmonic imaging studies were stored on a magneto-optical disk for off-line analysis. For harmonic imaging $4 \mathrm{~g}$ of a galactose based ultrasound contrast agent (Levovist ${ }^{\circledR}$, Schering AG, Berlin, Germany) was used in a concentration of $400 \mathrm{mg} / \mathrm{ml}$. The ultrasound contrast agent was given as an intravenous bolus by an infusion pump at a rate of $2.5 \mathrm{ml} / \mathrm{s}$ into the antecubital vein, which was cannulated with an 18 gauge catheter.

\section{Harmonic imaging studies}

Results of cranial MRI or CT for all the patients were known to the sonographer. For ultrasound investigation the side ipsilateral to the tumour was chosen to keep depth dependent ultrasound attenuation to a minimum. Each examination was started in native B mode using the transtemporal approach and a transversal insonation plane. To ensure an adequate acoustic bone window a satisfactory depiction of the hyporeflective butterfly shaped brain stem in the mesencephalic plane of section, and of the third ventricle in the diencephalic plane, was obtained before locating the tumour. The ultrasound probe and insonation depth were then fitted to the largest depictable diameter of the tumour, requiring that the midline and either the brain stem or the thalamus was visible in the same plane of section as healthy reference ROI. If the tumour was not detectable in native $\mathrm{B}$ mode imaging, the probe was adjusted to the assumed localisation according to the magnetic resonance or CT images in the corresponding insonation plane. Before switching the quickset to the acoustic quantification mode to start the harmonic examination, depth gain compensation was adjusted individually. The acoustic quantification mode provides an integrated on-line capability to measure the average acoustic image intensity within a user specified ROI and is based on integrated backscatter technology. This is a relative measure of the total ultrasonic energy reflected by a small volume of interrogated tissue. To enable off-line generation of time-intensity curves, the acoustic densitometry "AD T-INT" study type was used. In each examination 62 time triggered images were recorded at a frame rate of $0.4 \mathrm{~Hz}$, so that a whole examination lasted no more than three minutes. The ultrasound contrast agent was applied after three baseline images.

The acoustic densitometry unit was used for off-line data analysis. Time-intensity curves were calculated from two ROI with a sample area of $21 \times 21$ pixels each to quantify contrast intensity of the backscattered signal. The ROI was chosen according to the suspected tumour area from the B mode image. The time-intensity curve with the maximum detectable peak contrast intensity was chosen from up to three ROI. If the tumour was not visible in the B mode image, the ROI were placed according to MRI/CT information.

The reference ROI (healthy brain tissue) was located in either the thalamus or the brain stem, depending on the insonated plane.

In order to reduce artefacts and erratic graph movements from patient or ultrasound probe movements, the images were manually aligned if necessary. The baseline for each calculated time-intensity curve was set as the mean acoustic intensity of the first three images after ultrasound contrast agent administration.

\section{Statistical analysis}

Three time-intensity curve parameters- the peak intensity (PI) (dB), the time to peak intensity (TP) (s), and the positive gradient $(\mathrm{PG})(\mathrm{dB} / \mathrm{s})$, which is the peak intensity divided by the time to peak intensity (PI/TP) -were used for statistical analysis. These parameters were compared intraindividually between tumour and healthy tissue as well as interindividually between different tumour types. For the latter analysis, tumours were grouped into four tumour types: high grade gliomas, meningiomas, metastases, and others (tumours not classified under the first three headings). In addition to the three time-intensity curve parameters (PI, TP, and PG) the ratio of the PI of tumour and healthy brain tissue was compared between these subgroups.

The Wilcoxon test was used for intraindividual analyses, and the Mann-Whitney and Kruskal-Wallis tests for interindividual analyses, as appropriate. Differences were considered as statistically significant at probability $(\mathrm{p})$ values of $<0.05$.

\section{RESULTS}

\section{General}

Twenty nine patients with brain tumours underwent a screening ultrasound examination, of whom six where diagnosed as having a high grade glioma, nine a meningioma, seven metastases, and seven other tumours (three low grade gliomas, one chordoma, one neurinoma of the trigeminal nerve, and two tumours of the pituitary gland). Twenty seven of these patients were found to have an adequate acoustic bone 

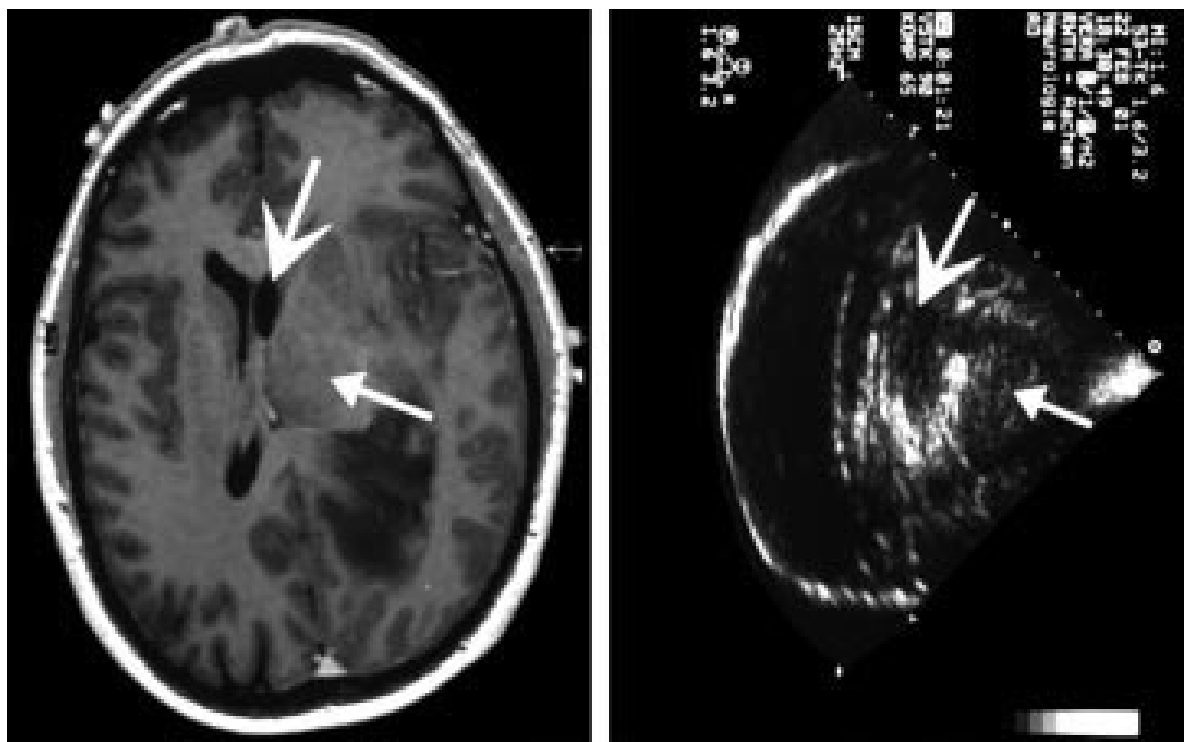

Figure 1 Magnetic resonance scan and native B mode image of a high grade glioma in corresponding transverse axial planes of section. The tumour (smaller arrow) is located laterally to the lateral ventricles (larger arrow). The B mode image shows an oval lesion with a heterogeneous echo that seems well defined from the surrounding tissue. The two low signal bands extending from the ultrasound probe towards the midline are artefacts caused by a heterogeneous temporal bone window.

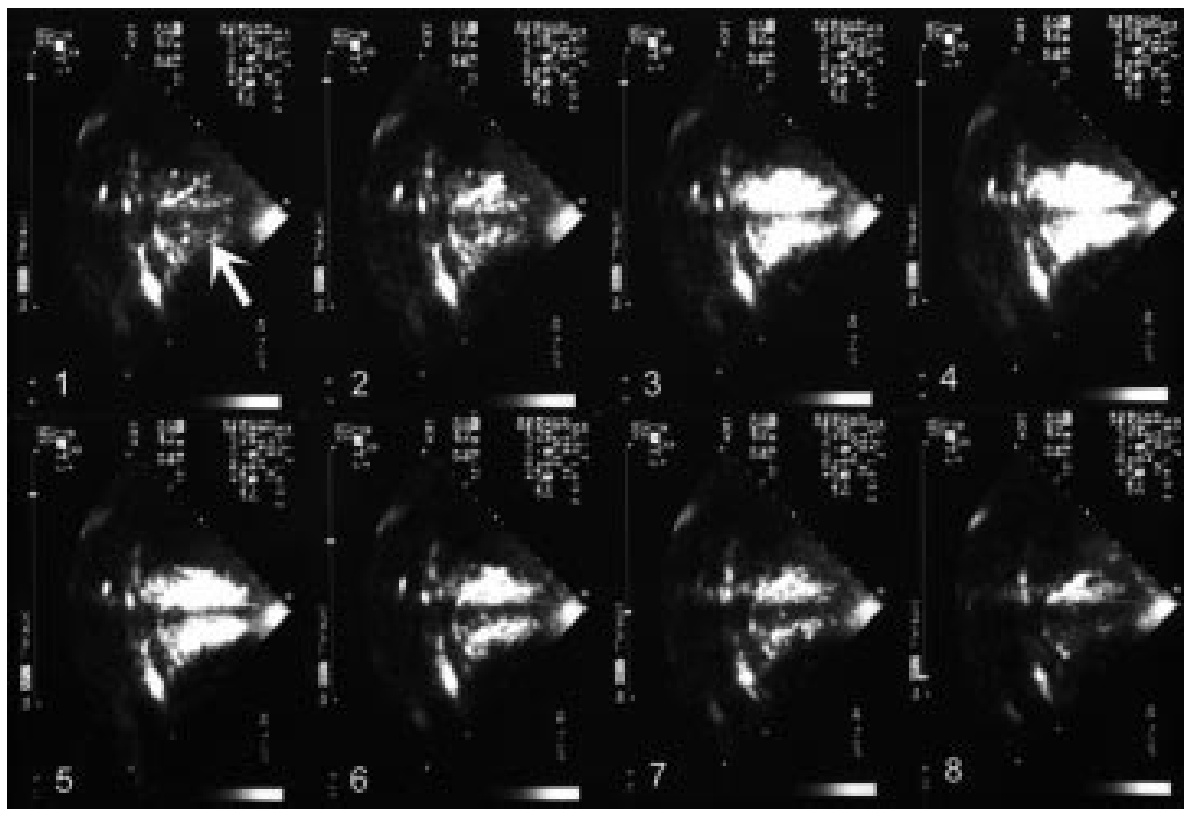

Figure 2 Second harmonic imaging examination of a high grade glioma (views numbered sequentially). The tumour (arrow) is identified easily before application of ultrasound contrast agent lateral to the thalamus; the pineal gland is strongly hyperechogenic. After application of ultrasound contrast agent, rapid and intense contrast enhancement can be visualised in the tumour (maximum in image 4), while other regions show only moderate enhancement. Contrast enhancement persists at the end of the examination (image 8). The low signal band extending from the ultrasound probe towards the midline is an artefact.

window and could therefore be included in the study; two with an insufficient temporal bone window were excluded. Both the latter had metastatic brain tumours. In 21 of the 27 patients the tumour was visible in native B scan, while in six native imaging failed to identify the lesion. Two of these patients had a rather low quality bone window, while in the other four the tumour was isoechogenic compared with the adjacent brain on native B scan. Figure 1 shows a native B scan and the corresponding magnetic resonance image of a high grade glioma. Signal intensity decreased in the harmonic mode but image quality was well sustained. In integrated backscatter mode, however, spatial resolution was usually lower. Contrast medium administration led to visibly detect- able contrast enhancement at least ipsilateral to the insonated side in all patients. The administration of the contrast agent was not associated with any side effects. Figure 2 shows an SHI examination of a high grade glioma (the same patient as in fig 1).

\section{Tumour versus healthy tissue}

After administration of the contrast agent, contrast enhancement was visible in the tumour region in all but two of the patients. The data are listed in table 1. Comparison of the analysed time-intensity curves of the designated ROI showed dramatic rises of contrast enhancement and high peak intensities in tumour tissue, while the curve calculated from 
Table 1 Peak intensity, time to peak intensity, and positive gradient in different regions of interest: tumour and brain tissue

\begin{tabular}{|c|c|c|c|c|c|c|}
\hline & $\mathrm{PI}(\mathrm{dB})$ & $\mathrm{p}$ Value & $\mathrm{TP}(\mathrm{s})$ & $\mathrm{p}$ Value & $P G(d B / s)$ & $p$ Value \\
\hline Tumour & $11.8(6.9)$ & \multirow{2}{*}{0.0001 * } & $37.1(32.3)$ & \multirow{2}{*}{$0.142^{*}$} & $0.69(0.71)$ & \multirow{2}{*}{0.001 * } \\
\hline Brain tissue & $5.1(2.2)$ & & $50.2(40.7)$ & & & \\
\hline
\end{tabular}

Table 2 Peak intensity, time to peak intensity, and positive gradient in different tumour types: high grade glioma, meningioma, metastases, and other tumours

\begin{tabular}{|c|c|c|c|c|}
\hline & $\mathrm{PI}(\mathrm{dB})$ & $\mathrm{TP}(\mathrm{s})$ & $P G(d B / s)$ & PI ratio \\
\hline $\begin{array}{l}\text { High grade glioma } \\
(n=6)\end{array}$ & $14.4(7.5)$ & 18.3 (12.5) & $1.1(7.8)$ & $2.7(1.0)$ \\
\hline Meningioma $(n=9)$ & $11.9(8.4)$ & 39.6 (32.7) & $0.64(0.70)$ & 2.5 (1.7) \\
\hline Metastases $(n=5)$ & $10.4(4.7)$ & $36.7(26.2)$ & $0.70(0.94)$ & $3.1(2.5)$ \\
\hline Other tumours $(n=7)$ & $10.5(6.2)$ & $50.2(44.2)$ & $0.43(0.42)$ & $2.3(2.4)$ \\
\hline \multicolumn{5}{|c|}{$\begin{array}{l}\text { Values are mean (SD). } \\
\text { The Kruskal-Wallis test was used for analysis and revealed no significant differences in any of the variables } \\
\text { between the four groups. } \\
\text { PG, positive gradient; PI, peak intensity; PI ratio, ratio of the peak intensity in tumor tissue to the peak } \\
\text { intensity in healthy tissue; TP, time to peak intensity. }\end{array}$} \\
\hline
\end{tabular}

healthy tissue tended to have a more steady increase and lower peaks, which resulted quantitatively in significantly higher peak intensities and positive gradients in the tumour compared with healthy tissue in all patients (mean PI, $11.8 \mathrm{v}$ $5.1 \mathrm{~dB}$; mean PG, $0.69 v 0.16 \mathrm{~dB} / \mathrm{s} ; \mathrm{p}=0.0001$ and $\mathrm{p}=0.001$, respectively). Mean time to peak intensity was noticeably shorter in tumour tissue, but the difference was not statistically significant $(37.1 \mathrm{~s}$ in tumour $v 50.2 \mathrm{~s}$ in healthy tissue; $p=0.14$ ). Both peak intensities and positive gradients had a considerably wider range in tumour than in healthy tissue, ranging from $2.2 \mathrm{~dB}$ to $25.8 \mathrm{~dB}$ and from $0.03 \mathrm{~dB} / \mathrm{s}$ to 2.34 $\mathrm{dB} / \mathrm{s}$, compared with $2.0 \mathrm{~dB}$ to $10.3 \mathrm{~dB}$ and $0.03 \mathrm{~dB} / \mathrm{s}$ to 0.90 $\mathrm{dB} / \mathrm{s}$ in healthy tissue. In contrast, time to peak intensity showed a similar range in tumour tissue as in healthy tissue (3.7 to 138.8 s $v 13.7$ to $147.7 \mathrm{~s}$, respectively).

\section{Benign versus malignant tumours}

Fifteen of the 27 patients had a benign tumour and 12 had a malignant tumour. Comparison of the time-intensity curve parameters between these two groups showed no significant differences in peak intensity and positive gradient, although there was a noticeable trend for malignant tumours to show higher positive gradients with shorter times to peak intensity. Thus while the peak intensity reached a mean of $13.7 \mathrm{~dB}$ in the malignant tumours and $10.3 \mathrm{~dB}$ in the benign tumours, the mean positive gradient was $0.98 \mathrm{~dB} / \mathrm{s}$ in the malignant group compared with $0.46 \mathrm{~dB} / \mathrm{s}$ in the benign group $(\mathrm{p}=0.057)$, and the mean times to peak intensity were $25.8 \mathrm{~s} v 46.1 \mathrm{~s}$, respectively $(p=0.084)$.

\section{Tumour groups}

The four tumour groups (high grade gliomas, meningiomas, metastases, and other tumours) consisted of approximately the same numbers of patients. Table 2 gives an overview of the data for the four groups. Comparison of the time-intensity curves showed considerably stronger and faster contrast enhancement in high grade gliomas, with higher peak intensities and positive gradients as well as shorter times to peak intensity. However, there were no statistically significant differences between the four groups. Mean PI was $14.4 \mathrm{~dB}$ in high grade gliomas, $11.9 \mathrm{~dB}$ in meningiomas, $10.4 \mathrm{~dB}$ in metastases, and $10.5 \mathrm{~dB}$ in the mixed group $(\mathrm{p}=0.82)$. Mean
TP was $18.3 \mathrm{~s}$ in high grade gliomas, $39.6 \mathrm{~s}$ in meningiomas, $36.7 \mathrm{~s}$ in metastases, and $50.2 \mathrm{~s}$ in the mixed group. PG varied from $1.11 \mathrm{~dB} / \mathrm{s}$ in high grade gliomas, to $0.64 \mathrm{~dB} / \mathrm{s}$ in meningiomas, $0.70 \mathrm{~dB} / \mathrm{s}$ in metastases, and $0.43 \mathrm{~dB} / \mathrm{s}$ in the last group $(p=0.36)$. Mean ratios of the peak intensity of tumour to healthy tissue were highest in metastases and tended to be higher in high grade gliomas than in the other two groups, but these differences also did not reach statistical significance (high grade glioma, 2.7; meningiomas, 2.5; metastases, 3.1; others, $2.3 ; \mathrm{p}=0.72$ ).

\section{DISCUSSION}

Harmonic imaging of the human brain was introduced only a few years ago and has proved reliable in examining human brain perfusion and assessing several perfusion indices in healthy individuals as well as perfusion disturbances in acute ischaemic stroke. ${ }^{8-11}$ We therefore took advantage of the potential of this new ultrasound technique to depict and assess brain tumour perfusion. To our knowledge, this is the first study on second harmonic imaging of human brain tumours. Given that the number of patients investigated was small and the number of tumour types even smaller, the conclusions drawn from our data should be regarded as preliminary and viewed with reservation. Nevertheless the study has for the first time shown the possibility of assessing brain tumour perfusion by means of transcranial SHI. With known tumour location from CT and MRI, the tumour detection rate in native B scan was about $75 \%$, increasing to $93 \%$ in contrast harmonic mode. This is comparable with a previous study on native transcranial sonography of brain tumours in the adult, where the rate of tumour identification was $80 \%$ when radiological findings were known to the investigators. ${ }^{25}$ Application of microbubble specific imaging modes clearly increase the sensitivity of ultrasound to brain tumours, as has been shown in the case of focal liver masses, where lesions of less than 1 $\mathrm{cm}$ diameter can be detected..$^{17}{ }^{18}$

Analysis of the time-intensity curves reflected the higher blood flow in the tumour, showing significantly higher peak intensities and positive gradients in all patients compared with healthy tissue. We conclude that SHI allows reliable identification of hyperperfused tissue and satisfactory differentiation from normally perfused tissue. The wide range of 
peak intensities and consequently of positive gradients in the tumour tissue (2.2 to $25.8 \mathrm{~dB}$ and 0.03 to $2.34 \mathrm{~dB} / \mathrm{s}$, compared with 2.0 to $10.3 \mathrm{~dB}$ and 0.03 to $0.90 \mathrm{~dB} / \mathrm{s}$ in healthy tissue) may reflect the variability of tumour vascularisation and consequently of perfusion.

This variability is again reflected in the comparative analysis of time-intensity curve parameters between benign and malignant tumours. There was a noticeable but nonsignificant trend towards shorter times to peak intensity and positive gradients in the malignant tumours (mean positive gradient, $0.98 \vee 0.46 \mathrm{~dB} / \mathrm{s}, \mathrm{p}=0.057$; mean time to peak intensity, $25.8 v 46.1 \mathrm{~s}, \mathrm{p}=0.084)$. This trend is explicable by the vasculature of high grade gliomas which formed part of the malignant group, typically consisting of various types of vessel, vessel meshworks, and arteriovenous anastomoses that allow rapid blood flow and lead to especially high peak intensities and positive gradients, accompanied by remarkably short times to peak intensity. ${ }^{24}$ However, it is unlikely that a larger number of patients would have led to significant differences between benign and malignant tumours, because the benign group included a particularly well perfused tumourthe meningioma. A recent cerebral blood flow study evaluating different brain tumours using xenon CT showed significantly higher blood flows in meningiomas than in gliomas or neurinomas. ${ }^{28}$ In our rather small groups we found that meningiomas had the second highest mean peak intensity of contrast enhancement after high grade gliomas ( $11.9 \mathrm{~dB}$ and 14.4 $\mathrm{dB}$ ), probably representing the high blood flow in these tumours, while metastases and other tumour types reached between 10.4 and $10.5 \mathrm{~dB}$. While the mean time to peak intensity is again much shorter and the positive gradient rather higher in high grade gliomas, differences in these indices between the other groups were not as marked, and meningiomas, in particular, had rather similar times to peak intensity and similar positive gradients when compared with metastases. In comparison, the mixed tumour group showed the lowest values for these indices. Comparison of peak intensity ratios of tumour tissue to healthy tissue between the different tumour types did not seem to be valuable, as all ratios were quite similar.

Larger numbers of patients will have to be studied to allow full analysis of the parameters examined here. Further investigation may well lead to the introduction of other useful parameters, especially with regard to contrast washout. This should allow the evaluation of contrast kinetics through analysis of different time-intensity curve parameters in order to explore non-invasive healthy differentiation and grading. Taking into consideration that a first generation contrast agent was used in this study, further research is needed to compare different ultrasound contrast agents with varying stabilities and kinetics. ${ }^{29}{ }^{30}$ Additional research is required on specific enhancement patterns that are known from focal liver lesions $^{31}$-liver mass evaluation with ultrasound relies strongly on lesion characterisation by typical and reproducible features such as rim enhancement in metastases, or peripheral enhancement with centripetal extension in haemangiomas, similar to changes in the enhancement pattern found in spiral CT. ${ }^{17}{ }^{18}$ Future studies should therefore try to correlate parameters obtained by ultrasound perfusion imaging with the known parameters of other perfusion imaging techniques, such as MRI or CT perfusion imaging. ${ }^{23} 24$ Analysis of healthy vasculature should be studied, including the ranges of morphological and pathophysiological characteristics of healthy vessels and their grading, as this would allow the application of the specific properties that ultrasound perfusion imaging offers.

\section{Conclusions}

Our study shows for the first time that second harmonic imaging of brain tumours is not only feasible but may also be beneficial in terms of providing additional and alternative information about healthy vasculature and perfusion. In comparison with other imaging modes, SHI provides a rapid, practical, and cost-effective bedside technique, particularly for critically ill patients and when multiple follow up investigations are needed. Further studies are necessary to evaluate the clinical potential of SHI in brain tumours and its role as a diagnostic and follow up procedure for patients undergoing surgical treatment, radiation, or chemotherapy, where it may prove to be a useful new tool for evaluating the effects of treatment on healthy vasculature and for the improved detection of recurrent disease. Comparison with neuroradiological and neuropathological findings is now necessary to establish this new technique among current imaging resources.

\section{ACKNOWLEDGEMENTS}

We thank Prof Dr K Willmes-v Hinckeldey for his help with statistical analysis and Dr Stuart Fellows for his thoughtful review of the manuscript.

\section{Authors' affiliations}

J U Harrer, C Klötzsch, Department of Neurology, University Hospital Aachen, Aachen, Germany

L Mayfrank, Department of Neurosurgery, University Hospital Aachen

M Mull, Department of Neuroradiology, University Hospital Aachen

Competing interests: none declared

\section{REFERENCES}

1 De Jong N, Ten Cate FJ, Lancee CT, et al. Principles and recent developments in ultrasound contrast agents. Ultrasonics 1991:29:324-30.

2 Schrope B, Newhouse VL. Second harmonic blood perfusion measurement. Ultrasound Med Biol 1993;19:567-79.

3 Burns PN. Harmonic imaging with ultrasound contrast agents. Clin Radiol 1996;51:50-5.

4 Frinking PJA, Bouakaz A, Kirkhorn J, et al. Ultrasound contrast imaging: current and new potential methods. Ultrasound Med Biol 2000;26:965-75

5 Porter TR, Li S, Kricsfeld D, et al. Detection of myocardial perfusion in multiple echocardiographic windows with one intravenous injection of microbubbles using transient response second harmonic imaging. J Am Coll Cardiol 1997:29:791-9.

6 Hancock J, Dittrich $\mathrm{H}$, Jewitt DE, et al. Evaluation of myocardial, hepatic and renal perfusion in a variety of clinical conditions using an intravenous ultrasound contrast agent (Optison) and second harmonic imaging. Heart 1999:81:636-41.

7 Wilson SR, Burns PN, Muradali D, et al. Harmonic hepatic US with microbubble contrast agent: initial experience showing improved characterization of hemangioma, hepatocellular carcinoma, and metastasis. Radiology 2000;215:153-61.

8 Postert T, Muhs A, Meves S, et al. Transient response harmonic imaging Stroke 1998;29:1901-7.

9 Federlein J, Postert T, Meves S, et al. Büttner T. Ultrasonic evaluation of pathological brain perfusion in acute stroke using second harmonic imaging. J Neurol Neurosurg Psychiatry 2000;69:616-22.

10 Seidel G, Meyer K. Harmonic imaging - a new method for the sonographic assessment of cerebral perfusion. Eur J Ultrasound $2001 ; 14: 103-13$.

11 Harrer JU, Klötzsch C. Second harmonic imaging of the human brain: The practicability of coronal insonation planes and alternative perfusion parameters. Stroke 2002;33:1530-5

12 Postert T, Federlein J, Rose J, et al. Ultrasonic assessment of physiological echo-contrast agent distribution in brain parenchyma with transient response harmonic imaging. J Neuroimaging 2001;11:18-24

13 D'Sa A. Acoustic densitometry. Andover, MA: Hewlett-Packard Co, 1999.

14 Foster FS, Burns PN, Simpson DH, et al. Ultrasound for the visualization and quantification of tumor microcirculation. Cancer and Metastasis reviews 2000;19:131-8.

15 Heckemann RA, Cosgrove DO, Blomley M, et al. Liver lesions: intermittent second-harmonic gray-scale US can increase conspicuity with microbubble contrast material - early experience. Radiology 2000;21 6:592-6

16 Burns PN, Wilson SR, Simpson DH. Pulse inversion imaging of liver blood flow: improved method for characterizing focal masses with microbubble contrast. Invest Radiol 2000;35:58-71.

17 Wilson SR, Burns PN. Liver mass evaluation with ultrasound: the impact of microbubble contrast agents and pulse inversion imaging. Semin Liver Dis 2001;21:147-59.

18 Bloomley MJK, Cooke JC, Unger EC, et al. Microbubble contrast agents: a new era in ultrasound. BM 2001;322:1222-5. 
19 Gillard JH, Minhas PS, Hayball MP, et al. Assessment of quantitative computed tomographic cerebral perfusion imaging with $\mathrm{H}_{2}(15) \mathrm{O}$ positron emission tomography. Neurol Res 2000;22:457-64.

20 Pomper MG, Port JD. New techniques in magnetic resonance imaging of brain tumors. Neuroimaging Clin N Am 2001;11:501-25.

21 Nelson SJ. Analysis of volume MRI and MR spectroscopic imaging data for the evaluation of patients with brain tumors. Magn Reson Med 2001;46:228-239

22 Camargo EE. Brain SPECT in neurology and psychiatry. J Nucl Med 2001:42:611-23.

23 Lev $\mathrm{MH}$, Hochberg F. Perfusion magnetic resonance imaging to assess brain tumor responses to new therapies. Cancer Control 1998;5: 115-23

24 Sugahara T, Korogi $Y$, Kochi $M$, et al. Perfusion-sensitive MR imaging of gliomas: comparison between gradient-echo and spin-echo echo-planar imaging techniques. Am J Neuroradiol 2001;22:1306-15.

25 Meyer K, Seidel G, Knopp U. Transcranial sonography of brain tumors in the adult: an in vitro and in vivo study. J Neuroimaging 2001:11:287-92.
26 Becker G, Hofmann E, Woydt M, et al. Postoperative neuroimaging of high-grade gliomas: comparison of transcranial sonography, magnetic resonance imaging and computed tomography. Neurosurgery 1999;44:469-77.

27 Vordermark D, Becker G, Flentie M, et al. Transcranial sonography: integration into target volume definition for glioblastoma multiforme. Int $\rfloor$ Radiat Oncol Biol Phys 2000;47:565-71.

28 Mirzai S, Samii M. Current status and future challenges in cerebral blood flow mapping in intracranial tumors. Keio J Med 2000;49/suppl 1):A16-24.

29 Sboros V, Moran CM, Anderson T, et al. An in vitro comparison of ultrasonic contrast agents in solution with varying air levels. Ultrasound Med Biol 2000;26:807-18

30 Seidel G, Vidal-Langwasser M, Algermissen C, et al. The influence of Doppler system settings on the clearance kinetics of different ultrasound contrast agents. Eur J Ultrasound 1999;9:167-75.

$31 \mathrm{Kim}$ TK, Choi BI, Han JK, et al. Hepatic tumors: contrast agent enhancement patterns with pulse-inversion harmonic US. Radiology 2000;216:411-17.

\section{NEUROLOGICAL PICTURE}

\section{Muscle hypertrophy in myotonia congenita}

A 28 year old male presented to our neurology service with stiffness of a few years' duration in all muscles. The stiffness was maximum at the onset of exercise and his muscles loosened up after some time. He had no difficulty in releasing his grip after holding an object. He had very well developed muscles in spite of a sedentary lifestyle. There was no family history of similar problems. On examination he showed percussion myotonia. There was no grip myotonia or eyelid myotonia. Nerve conduction studies were normal. Electromyogram showed myotonic potentials with a characteristic dive bomber sound on the loudspeaker. A diagnosis of myotonia congenita was made. He most likely had the recessive form of myotonia congenita because both parents were unaffected clinically. He was started on Mexiletene 200 mg three times daily with resultant improvement in stiffness.

A dramatic increase in the bulk and definition of voluntary muscles is common in patients with myotonia congenita, presumably because the skeletal muscles are in an almost continuous state of muscle contraction. In most myotonias, repetitive contractions reduce muscle stiffness except for paramyotonia, where there is a paradoxica increase in stiffness with repetitive exercise.

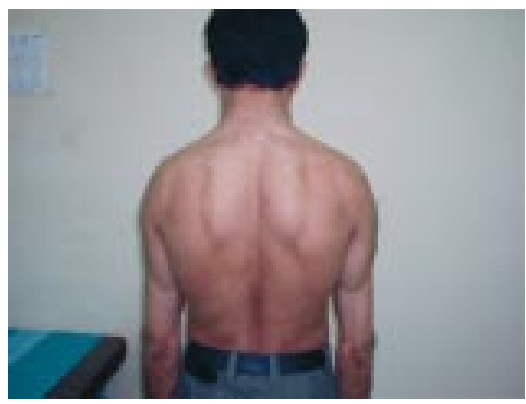

Myotonia, defined as delayed relaxation of muscle after contraction, is seen in a group of genetic disorders that includes autosomal dominant myotonia congenita (Thomsen's disease) and autosomal recessive myotonia congenita (Becker's disease). Both diseases are caused by mutations in the skeletal muscle voltage gated chloride channel gene. Becker's disease resembles Thomsen's disease but there are also differences, eg later onset and transient muscle weakness experienced by many patients during muscle exertion after rest. The criterion for the differential diagnosis of Thomsen or Becker type myotonia is the mode of inheritance. ${ }^{2}$

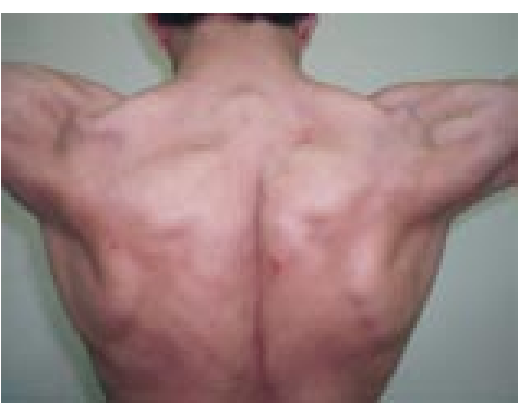

B Varkey, L Varkey

Indo-American Hospital, Chemmanakary, Vaikom, Kerala, India

Correspondence to: Dr B Varkey; bobvarkey@vsnl.com

\section{References}

1 Zhang J, George AL Jr, Griggs RC, et al. Mutations in the human skeletal muscle chloride channel gene (CLCN1) associated with dominant and recessive myotonia congenital. Neurology 1996;47:993-8.

2 Kuhn E. Myotonia congenital (Thomsen) and recessive generalized myotonia (Becker). Nervenarzt 1993;64:766-9. 